\title{
La «Charte» porte ses fruits
}

\author{
Hermann Amstad ${ }^{a}$, Christof Schmitz ${ }^{\text {, }}$, Peter Berchtold ${ }^{\mathrm{c}}$ \\ ${ }^{a}$ Dr méd., Secrétaire général ASSM, Berne; ${ }^{b}$ Dr rer. soc. oec., Directeur college M, Berne; ${ }^{c}$ PD Dr méd., Directeur college M, Berne
}

En novembre 2014, l'ASSM avait publié la Charte «Collaboration entre les professionnels de la santé». L’objectif de cette Charte était - et est - d'identifier le potentiel de la collaboration interprofessionnelle et de désigner les facteurs-clés qui y sont associés. L'ASSM a adopté diverses mesures pour accompagner la mise en œuvre de la Charte:

- En 2015, elle a fait établir un avis de droit afin de clarifier la portée de la responsabilité médicale.

- Au printemps 2016, elle a organisé à Zurich et à Morges des colloques de deux jours intitulés «Comment réussir l'interprofessionnalité?».

- En automne 2016, elle a mis au concours le Prix ASSM «Interprofessionnalité».

- En décembre 2016, elle a organisé le symposium "L'interprofessionnalité dans le système de santé: jusqu'ici - et ensuite?».

- Début 2017, elle a publié une étude concernant «La pratique d'une collaboration interprofessionnelle réussie».

\section{Avis de droit sur la responsabilité médicale}

La question de la portée de la responsabilité médicale a été régulièrement soulevée dans les débats publics autour de la «Charte». Un avis de droit, ${ }^{1}$ réalisé par l'Institut de droit de la santé de l'Université de Neuchâtel (IDS) à la demande de l'ASSM, répond à ces questions. L'avis de droit clarifie d'une part la notion de "responsabilité» et conclut d'autre part qu'en principe chaque professionnel de la santé endosse une responsabilité dans le cadre de son activité et en assume les conséquences.

La responsabilité pour les actes de tierces personnes dépend du statut de celles-ci. L'avis de droit distingue trois statuts et précise que plus la tierce personne est indépendante, plus elle doit assumer de responsabilités. Il va de soi que cette règle s'applique également à la collaboration interprofessionnelle.

L'avis de droit «Responsabilité médicale» est publié sur le site Internet de l'ASSM sous: assm.ch $\rightarrow$ Projets $\rightarrow$ Interprofessionnalité $\rightarrow$ Avis de droit. connaître de tels exemples, l'ASSM a mis au concours, en autome 2016, le Prix ASSM «Interprofessionnalité» pour des projets émanant de la pratique, de la formation et de la recherche. 42 requêtes ont été soumises suite à la mise au concours, 29 d'entre elles se sont vues décerner le prix. La remise des prix s'est déroulée début décembre, dans le cadre du symposium "L'interprofessionnalité dans le système de santé». Les exemples de bonne pratique honorés proviennent de toutes les régions de la Suisse et de diverses organisations de soins, de formation et de recherche.

Certaines différences régionales sont frappantes: parmi les 11 projets de la Suisse romande, 7 d'entre eux proviennent du domaine de la formation et 4 de la pratique. En Suisse alémanique, la proportion est inversée: 6 projets du domaine de la formation et 9 de la pratique. Concernant les projets du domaine de la formation, cela pourrait signifier qu'il manque un vis-àvis aux facultés de médecine en Suisse alémanique, comme c'est le cas en Suisse romande avec les hautes écoles spécialisées de la santé.

Dans les projets concernant toute la Suisse, on remarque le groupe de travail interprofessionnel «Dossier électronique du patient». Huit associations professionnelles se sont réunies pour participer ensemble

Les exemples de bonne pratique font figure de modèles et peuvent apporter soutien et conseil lors de la mise en œuvre de l'interprofessionnalité.

à la réalisation de l'eHealth. Pour ce faire, ils ont dû surmonter divers obstacles tels que, par exemple, une terminologie divergente pour des processus similaires. Pour le succès de la collaboration interprofessionnelle, il est essentiel d'identifier et d'éliminer ces difficultés.

\section{Etude «La pratique d'une collaboration interprofessionnelle réussie»}

Ces dernières années, la collaboration interprofessionnelle a fait l'objet d'une attention accrue. Tandis que la CIP devient une exigence croissante, la question de savoir ce qu'elle signifie concrètement pour les praticiens reste en suspens. Face à cette situation, l'ASSM a chargé le college $M$ de Berne d'une étude, dont l'objectif 
est de comprendre ce que signifie la CIP dans la pratique et d'en tirer des conclusions générales. L'institut de sociologie de l'Université Ludwig Maximilian de Munich a participé à cette étude. Les résultats sont publiés dans la série "swiss academies reports" et peuvent être téléchargés sur le site Internet de l'ASSM. ${ }^{2}$ Cette étude englobait cinq différents settings de soins: la prise en charge de base, les soins palliatifs, la chirurgie, la médecine interne et la psychiatrie. 25 entretiens ont été menés avec des experts de différents groupes professionnels des cinq settings mentionnés. La question de savoir ce que les praticiens considèrent comme CIP réussie ou comme CIP échouée était au cœur de ces entretiens, qui ont permis de déduire des stratégies pour l'amélioration de la collaboration des professionnels de la santé.

\section{La collaboration interprofessionnelle et la manière dont elle est perçue par les personnes concernées dépend largement des contextes ou des settings dans lesquels ces professionnels exercent.}

Les descriptions des personnes sondées montrent que la collaboration interprofessionnelle et la manière dont elle est perçue par les personnes concernées comme un succès ou comme un échec - dépend largement des contextes ou des settings dans lesquels ces professionnels exercent. L'étude permet en particulier de conclure que les exemples de CIP mentionnés comme réussis présentent des densifications respectivement des synchronisations intensifiées des processus et des activités de travail. L'étude a pu identifier trois modes de collaboration entre les professions.

- La densification coordinatrice concerne des formes de collaboration dans lesquelles des modes d'action clairement définis et bien établis au sein de l'institution interagissent avec des compétences éprouvées. En mode coordinateur, la collaboration entre différentes professions est souvent caractérisée par un manque de temps dû à une situation de crise ou par un horizon temporel clairement défini (par exemple réanimation, intervention chirurgicale). Cette forme de densification permet notamment d'accéder à des compétences éprouvées qui sont réunies en une sorte de "programme». Les programmations et les orientations de l'action obéissent en premier lieu à la rationalité médicale. Du point de vue de la compétence médicale, toutes les compétences professionnelles sont associées de manière complémentaire. Des exemples de ce type de densification ont notamment été observés dans les do- maines de la chirurgie et de la médecine interne. Le programme sert alors de modèle supérieur.

- La densification co-créative englobe des formes de collaboration qui misent sur la succession et la juxtaposition des différentes compétences professionnelles et individuelles, adaptées les unes aux autres, dans un horizon temporel relativement vaste (par exemple dans les soins palliatifs). Les différentes professions doivent rechercher ensemble des solutions et prendre des décisions d'un commun accord avec le patient. Aucune profession ne peut prétendre intrinsèquement à une quelconque domination. Les asymétries et les compétences décisionnelles dépendent de leurs échanges et de leurs mises à l'épreuve dans la pratique quotidienne. Dans la théorie d'organisation, la collaboration co-créative pourrait être décrite comme un modèle de réseau - avec toutes ses formes de monopole d'interprétation respectives.

- Entre la densification coordinatrice et la densification co-créative, il existe une large zone pouvant être qualifiée de densification type projet dans l'étude. Les CIP réussies peuvent être considérées comme des projets de collaboration intensifiée temporaires ou organisés de manière complexe. On peut citer comme exemples des tables rondes en médecine de premier recours, des tumorboards ou des gestions de cas interprofessionnelles ainsi que des formes d'organisation plus complexes au sens d'une relation réciproque attentionnée (heedful interrelating) en psychiatrie. Du point de vue de la théorie d'organisation, on part d'un modèle de noud.

Les descriptions des personnes interrogées expriment également dans quelle mesure (a) la médecine avec ses perceptions et ses compétences, respectivement l'individualité du patient, détient le monopole d'interprétation; (b) les compétences professionnelles spécifiques et l'individualité des professionnels de la santé entrent en jeu ou le dirige. A cet égard, la réanimation et les soins palliatifs s'opposent de manière exemplaire: d'un côté, la collaboration obéit à des logiques d'action purement médicales et, de l'autre côté, elle s'articule autour de processus de discussion pluridimensionnels entre la perspective des patients et les différentes perspectives professionnelles. Manifestement, le rapport entre ces deux dimensions peut varier. Le tableau 1 montre la relation entre les trois formes de densifications et le rapport médecine-individualité.

Létude conclut que les densifications de la collaboration interprofessionnelle - selon les perceptions et les 


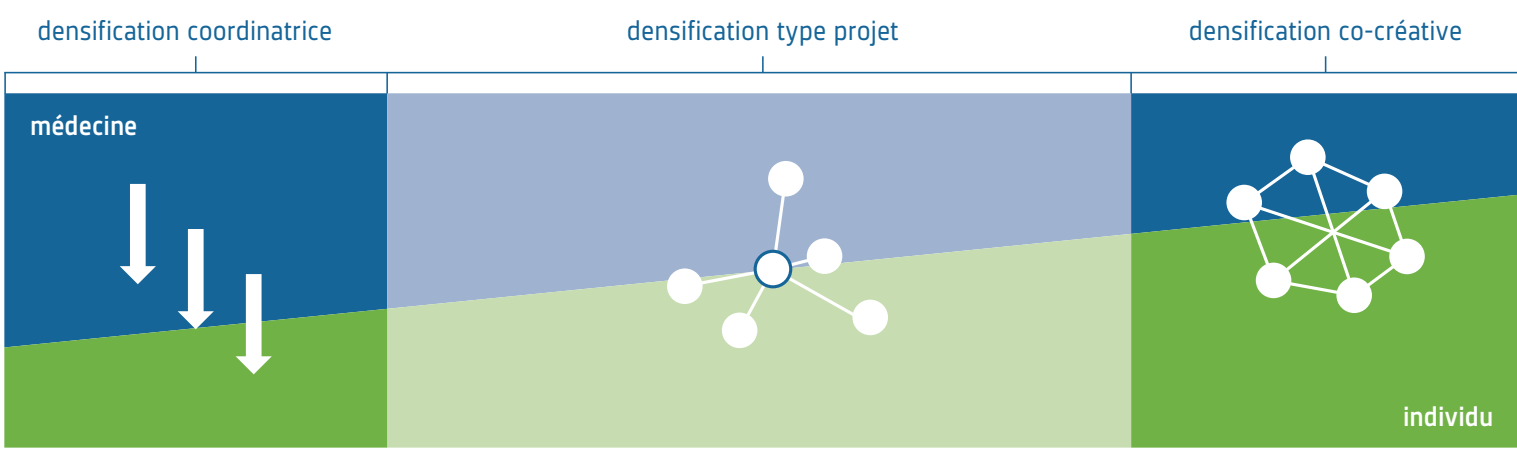

Figure 1: Collaboration interprofessionnelle: formes de densification, rapport médecine-individualité et mode d'organisation.

récits des spécialistes interrogés - se produisent en premier lieu autour des crises des patients. Ce faisant, les types de crise et les formes de densification de la collaboration interprofessionnelle ainsi provoquées divergent fortement. Les expertises des différents spécialistes coordonnent les crises somatiques aiguës selon une logique médicale; les crises de la fin de vie d'un patient en soins palliatifs demandent à chaque fois une thérapie individuelle, sans se soumettre à une procédure unique.

\section{Une culture constructive de la collaboration et un niveau d'égalité entre les différents groupes professionnels sont incontestablement des conditions préalables importantes à une CIP réussie.}

Tandis que ces deux formes de densifications se constituent autour de cas concrets, respectivement de patients, la troisième forme - la densification type projet dépend moins de la situation. Il s'agit de processus à l'organisation ponctuelle ou globale qui permettent un meilleur suivi des patients traités par plusieurs disciplines et professions. La densification type projet entre en jeu lorsqu'on ne veut pas se satisfaire d'une juxtaposition de compétences complémentaires faiblement coordonnées - le «cas normal» de la prise en charge médicale. Les densifications type projet varient énormément et se retrouvent dans de multiples settings.

\section{Conclusion et perspectives}

Une culture constructive de la collaboration et un niveau d'égalité entre les différents groupes professionnels sont incontestablement des conditions préalables importantes à une CIP réussie - et cela a été confirmé expressément par toutes les personnes interrogées. Parallèlement, les résultats de l'étude présentés ci-dessus montrent clairement qu'un changement culturel est nécessaire, sans toutefois être un point d'ancrage suffisant pour l'encouragement de la collaboration interprofessionnelle. De même, la reconnaissance et l'harmonisation des exigences spécifiques aux différents settings, définis du point de vue organisationnel et professionnel, sont tout aussi capitales.

Deuxièmement, si nous voulons prendre au sérieux les expériences des professionnels interrogés, la CIP ne doit pas correspondre à une (nouvelle) répartition des tâches entre les groupes professionnels ou à une délégation ou une substitution. Le transfert des tâches entre les groupes professionnels - aussi indiqué puisset-il être pour diverses raisons - n'influence pas systématiquement le moment de la densification des procédures et des actions tel qu'il est décrit. Reste néanmoins à signaler que la thématisation et l'implémentation de ces nouvelles répartitions peuvent être d'excellentes occasions de se livrer à des réflexions pertinentes pour la CIP.

Crédit illustration

Cf. note de bas de page 2 . 